\title{
Endocrinological Abnormalities Are a Main Feature of 17p13.1 Microduplication Syndrome: A New Case and Literature Review
}

\author{
Ilenia Maini ${ }^{a}$ f Ivan Ivanovski ${ }^{a}$ g Alessandro lodice ${ }^{b}$ Simonetta Rosato ${ }^{a}$ Marzia Pollazzon $^{a}$ \\ Manuela Mussini ${ }^{a} \quad$ Elga F. Belligni ${ }^{\text {h }}$ Charles Coutton ${ }^{j}$ Maria Marinellic Veronica Barbieri $^{c}$ \\ Manuela Napoli ${ }^{d}$ Rosario Pascarella ${ }^{d}$ Chiara Sartorie Francesca Madia ${ }^{i}$ Carlo Fusco $^{c}$

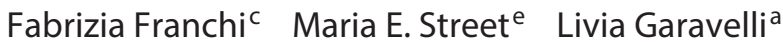 \\ ${ }^{a}$ Clinical Genetics Unit, bPaediatric Neuropsychiatry Unit, 'Medical Genetics Laboratory, Department of Obstetrics and \\ Paediatrics, dNeuroradiology Unit, Department of Diagnostic Imaging, e'Division of Paediatric Endocrinology and Diabetology, \\ Department of Obstetrics and Paediatrics, Arcispedale Santa Maria Nuova-IRCCS, Reggio Emilia, fScuola di Specializzazione in \\ Neuropsichiatria Infantile, Università degli Studi di Parma, Parma, 9Department of Surgical, Medical, Dental and Morphological \\ Sciences with interest in Transplant, Oncology and Regenerative Medicine, University of Modena and Reggio Emilia, Modena, \\ hDepartment of Paediatrics, University of Turin, Turin, and 'Laboratory of Neurogenetics and Neuroscience, Department of \\ Neuroscience, Istituto 'G. Gaslini', Genova, Italy; 'Laboratoire de Génétique Chromosomique, Département de Génétique et \\ Procréation, Hôpital Couple Enfant, Grenoble, France
}

\section{Established Facts}

- To date, 5 cases of 17p13.1 microduplications have been described in the literature.

- Intellectual disability is the only feature present in all 17p13.1 microduplication syndrome cases reported so far.

\section{Novel Insights}

- Endocrinological abnormalities such as obesity and diabetes have not been adequately stressed as important features of 17 p13.1 microduplication syndrome in previous reports.

\section{Key Words}

Diabetes · Endocrine disorders · Intellectual disability ·

Microduplication syndrome 17p13.1 - Obesity

\begin{abstract}
To date, 5 cases of 17p13.1 microduplications have been described in the literature. Intellectual disability was reported as the core feature, together with minor facial dysmorphisms and obesity, but a characteristic phenotype for 17p13.1 microduplication has not been delineated. Here, we describe a patient with a 1.56-Mb de novo duplication in 17p13.1, af-
\end{abstract}

fected by mild intellectual disability, facial dysmorphisms, obesity, and diabetes. By comparing the different phenotypes of currently described cases, we delineated the main clinical features of 17p13.1 microduplication syndrome. All patients described to date had variable facial dysmorphisms; therefore, it was difficult to define a common facial gestalt. Furthermore, we stress endocrinological abnormalities as important features and the need to monitor these over time.

(c) 2016 S. Karger AG, Basel

\section{KARGER}

E-Mail karger@karger.com www.karger.com/msy (c) 2016 S. Karger AG, Basel

$1661-8769 / 16 / 0076-0337 \$ 39.50 / 0$
Livia Garavelli

Clinical Genetics Unit, Department of Obstetrics and Paediatrics Arcispedale Santa Maria Nuova-IRCCS, Viale Risorgimento 80 IT-42123 Reggio Emilia (Italy)

E-Mail garavelli.livia@asmn.re.it 
The short arm of chromosome 17 is associated with a large variety of copy number losses and gains, resulting from nonallelic homologous recombinations between low copy repeats, that lead to a number of well-characterized genomic disorders. To date, few cases of $17 \mathrm{p} 13.1 \mathrm{mi}-$ croduplication have been reported [Belligni et al., 2012; Coutton et al., 2012; Kuroda et al., 2014; Mooneyham et al., 2014], but a distinct phenotype has not yet been defined. Intellectual disability was observed in all patients as the hallmark feature. In addition, a wide range of clinical signs have been reported such as behavioral anomalies, dysmorphisms, obesity, and endocrinological abnormalities. On the contrary, the corresponding $17 \mathrm{p} 13.1$ microdeletion syndrome is well described, and its relationship with neurodevelopmental delay was established [Komoike et al., 2010; Carvalho et al., 2014]. Indeed, it has been proposed that alteration of dosage-dependent genes may be involved in the genesis of neurodevelopmental disorders in patients with overlapped microdeletions and microduplications of 17p13.1 [Belligni et al., 2012; Coutton et al., 2012; Mooneyham et al., 2014]. Comparing the genetic profiles of cases of 17 p13.1 microduplications published up to 2014, Mooneyham et al. [2014] identified a common region of overlap including the DLG4, GABARAP, and CTDNEP1 genes as the candidate genes for intellectual disability in these patients.

Here, we describe a male patient with a 17p13.1 microduplication who, along with intellectual disability, facial dysmorphisms and obesity, presented with some previously unreported characteristics such as synoprhys, short hyperconvex nails, stiffness of interphalangeal hand joints, and motor dyspraxia. Specifically, we underline that endocrinological abnormalities are important features which have to be researched and closely monitored. Moreover, we attempted to describe a common clinical phenotype for $17 \mathrm{p} 13.1$ microduplication syndrome comparing the clinical and genetic aspects of the cases currently described.

\section{Patient and Methods}

\section{Clinical Report}

The propositus is a 13-year-old boy. He is the second child of healthy unrelated parents of Chinese ancestry. The family history of intellectual disability, diabetes, obesity or other endocrine conditions was negative. He was born at 38 weeks' gestation by spontaneous vaginal delivery after an uneventful pregnancy, and he weighed 2,700 g. Early neonatal adaptation was normal. During infancy and early childhood, the boy presented psychomotor developmental delay. His speech onset was at 3 years of age and was limited to a few words and simple sentences with some speech im-
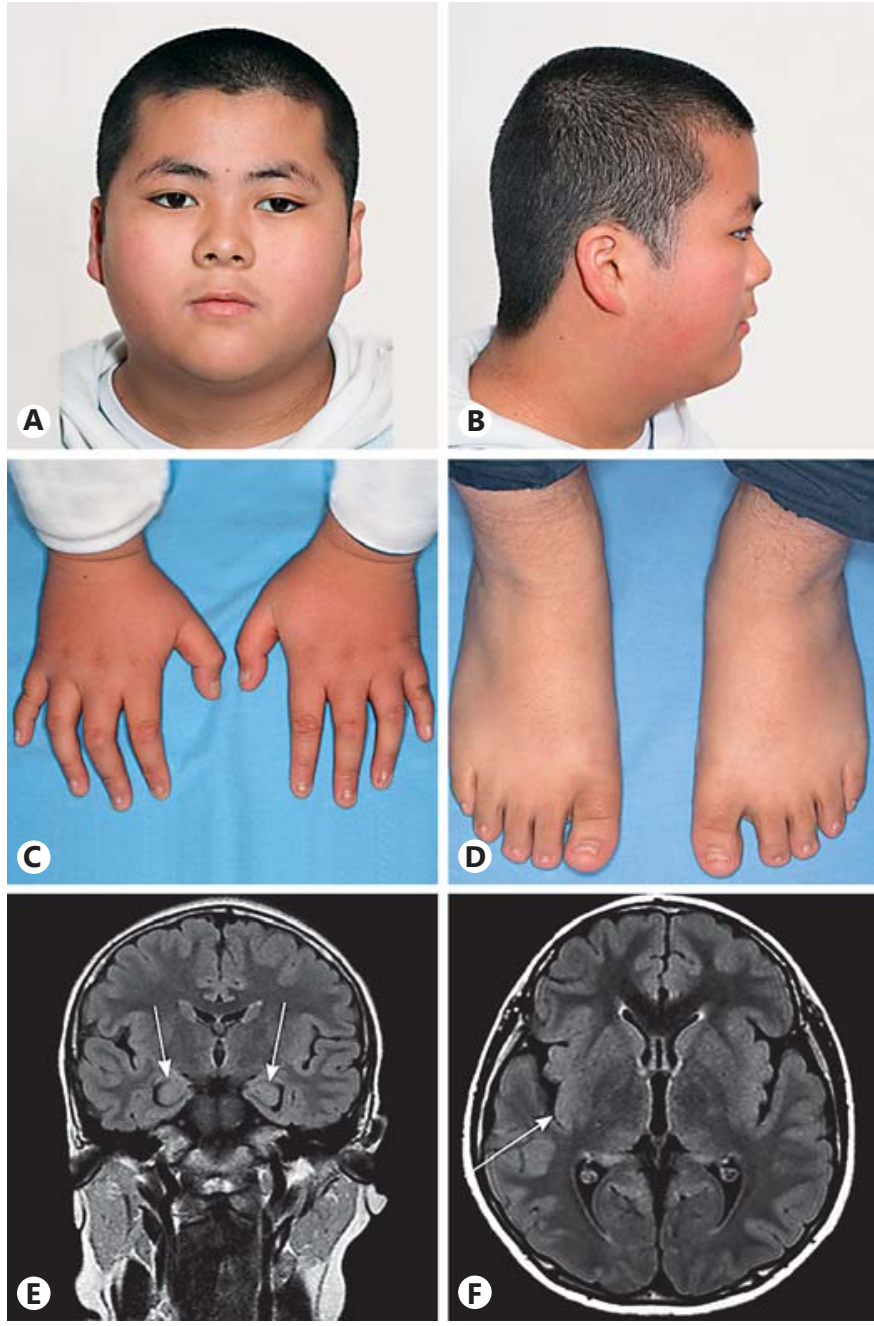

Fig. 1. The patient at 13 years of age. A, B Facial phenotype with a flat occiput, low posterior hairline, rounded face, synophrys, upslanting palpebral fissures, small nose with upturned nostrils, micrognathia, and slightly posteriorly rotated normally shaped ears. C Hands: tapering fingers with hyperconvex, hypoplastic fingernails. D Feet: brachydactyly, hypoplastic toenails. E Coronal FLAIR MRI of the brain shows bilateral hippocampal malrotation (arrows). F Axial FLAIR MRI of the brain shows reduced gyrification of insular cortex on the right posterior side (arrow).

pediments. At the age of 7 years, when the boy moved to Italy, mild intellectual disability (IQ 58) was documented.

Since infancy, food hoarding was observed which led to obesity. At the time of clinical evaluations, between 11 and 13 years of age, he showed marked hyperactivity and good social interaction skills despite communication difficulties and motor clumsiness.

At the age of 12 years, physical examination revealed: height $155 \mathrm{~cm}$ (75-90th percentile), weight $54 \mathrm{~kg}$ (97th percentile), and head circumference $54 \mathrm{~cm}$ (75-90th percentile). His BMI was 23.4, compatible with obesity [Cole et al., 2000]. Dysmorphic features included a flat occiput, low posterior hairline, rounded face, synophrys, upslanting palpebral fissures, a small nose with upturned nostrils, 


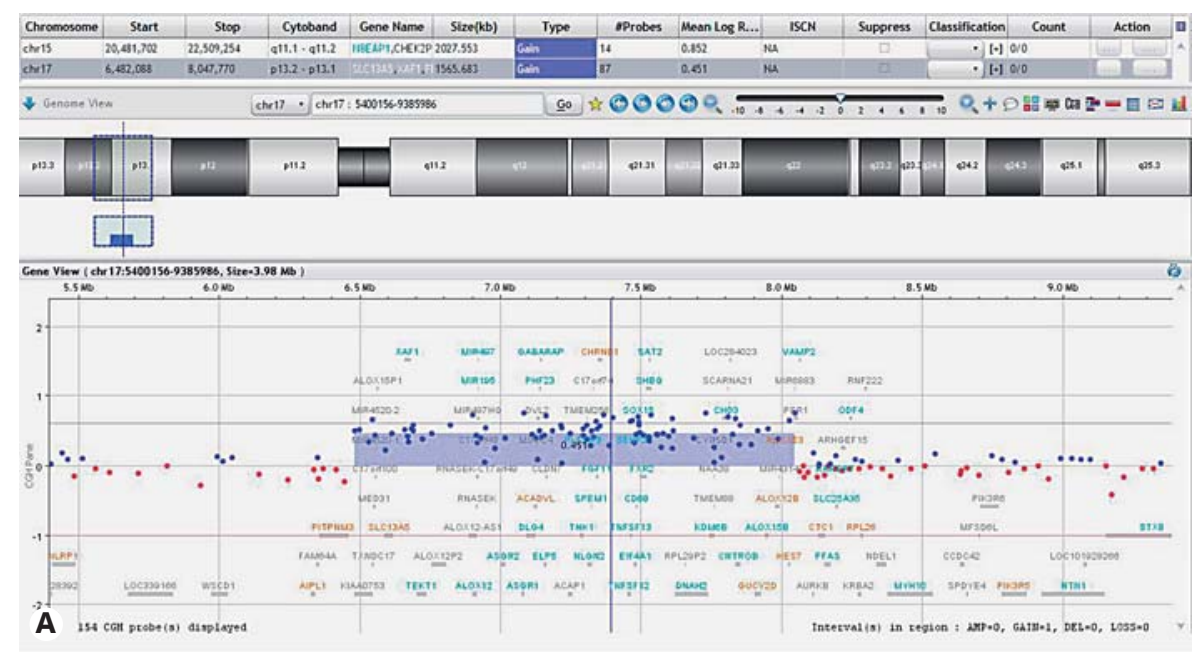

Fig. 2. Results of molecular cytogenetic investigations. A Array-CGH profile of chromosome 17 shows a $1.56-\mathrm{Mb}$ duplicated region in 17p13.1 (chr17: 6,482,088$8,047,770$ [h19]). The region harbors 89 RefSeq genes. B, C Results of FISH on interphase nuclei (B) and metaphase chromosomes (C) confirming a tandem $17 \mathrm{p} 13.1$ microduplication. TP53 (red) shows 2 signals on the interphase nuclei (arrows). Both signals are localized in chromosome $17 \mathrm{p}$ in the metaphase. The green signal shows the centromere region of chromosome 17 as a control (probe D17Z1).
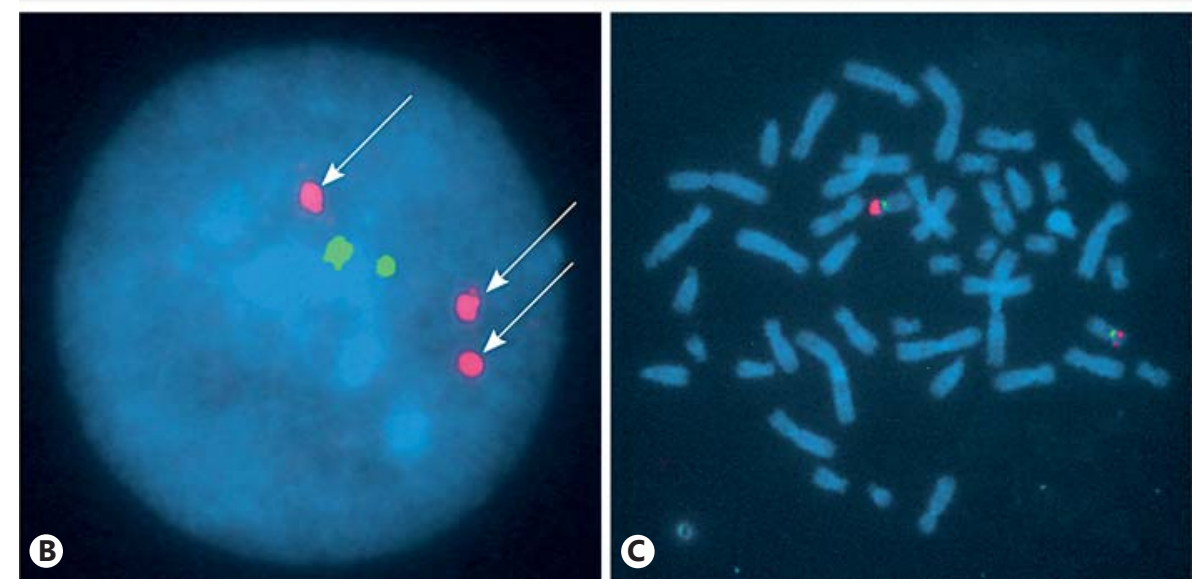

micrognathia, and slightly posteriorly rotated normally shaped ears (fig. 1A, B). His hands showed slight interphalangeal joint stiffness and tapering fingers with hyperconvex, hypoplastic nails (fig. 1C). He presented brachydactyly of the feet and hypoplastic toenails (fig. 1D). Neurological examination was normal except for motor dyspraxia.

Serial EEG recordings showed slow background activity without paroxysmal features. Brain MRI detected bilateral hippocampal malrotation (fig. 1E) and reduced gyrification of the insular cortex on the right posterior side (fig. 1F).

An ultrasound of the abdomen at 12 years showed a moderate hepatic fatty liver, with normal liver function tests compatible with nonalcoholic fatty liver disease. Other causes of liver disease were ruled out.

Endocrine and metabolic investigations showed increased hemoglobin A1c and borderline fasting blood glucose levels. Therefore, a 5-time point oral glucose tolerance test was performed revealing severe hyperinsulinism (peak $330.4 \mu \mathrm{IU} / \mathrm{ml}$ ) and glucose intolerance (blood glucose at $120 \mathrm{~min} 139.2 \mathrm{mg} / \mathrm{dl}$ ). Investigations for any autoimmune diseases were negative [Uwaifo et al., 2002]. A 6-month lifestyle intervention normalized the patient's hemoglobin A1c, and his BMI was reduced to 22.53 (overweight).

The boy's fundus oculi was normal, whereas the evaluation of his ocular motility documented prominent exotropia of the left eye. A chromosome analysis revealed a normal male karyotype.

17p13.1 Microduplication Syndrome

\section{Methods}

Array-CGH Analysis

Screening of genomic rearrangements was performed by arrayCGH using a Human Genome CGH Microarray kit $8 \times 60 \mathrm{~K}$ (Agilent Technologies, Santa Clara, Calif., USA) according to standard procedures. This analysis revealed a genomic duplication encompassing a 1,56-Mb region, from A_14_P132798 (6,482,088 bp) to A_14_P122419 (8,047,770 bp) (GRCh37/hg19 assembly) in chromosome 17p13.2p13.1. The region harbors 89 RefSeq genes. No additional aberrations were detected. Both parents were found to have a normal karyotype and $\mathrm{CGH}$ profile indicating that the duplication on chromosome 17 originated de novo in the son (fig. 2A).

\section{FISH Analysis}

FISH analysis using TP53 probe localized to 17p13.1 and probe $\mathrm{D} 17 \mathrm{Z1}$ for the centromeric region of chromosome 17 (Aquarius) was performed on interphase nuclei (fig. 2B) and metaphase chromosomes (fig. 2C) of peripheral blood cells samples obtained from the patient and parents following the manufacturer's protocol. The analysis confirmed the presence of a tandem duplication in chromosome 17. No duplications or translocations were present in his parents. FISH analysis of both parents was normal. 
Table 1. Clinical information for all cases carrying the 17p13.1 duplication

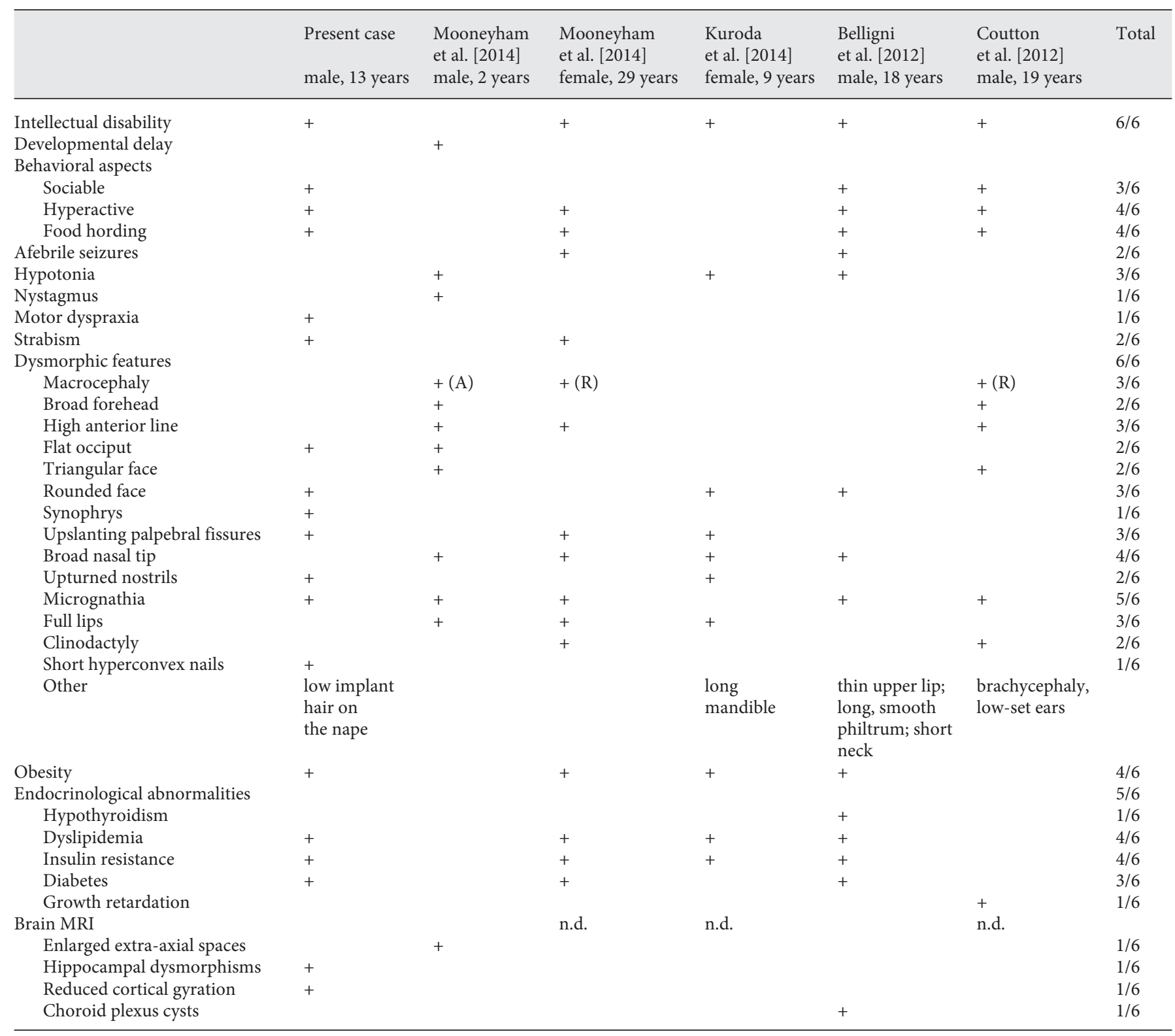

A = Absolute; $\mathrm{R}$ = relative; n.d. = not done; $+=$ present.

\section{Results and Discussion}

Five $17 \mathrm{p} 13.1$ microduplication cases have recently been described in the literature and were characterized by intellectual disability and other variable clinical signs [Belligni et al., 2012; Coutton et al., 2012; Kuroda et al., 2014; Mooneyham et al., 2014]. The first reported case was a 15 -year-old boy with moderate intellectual disability, non-febrile seizures, hypothyroidism, type 2 diabetes mellitus, hypertriglyceridemia, and astigmatism. His dysmorphic features included thin eyebrows, a bulbous nasal tip, long and smooth philtrum, malar hypoplasia, thin upper lip, micrognathia, and a short neck. Brain MRI detected choroid plexus cysts. A de novo duplication encompassing 790-830 kb $(7,016,453-7,807,789)$ in $17 \mathrm{p} 13.1$ was identified [Belligni et al., 2012].

The second case was a 4-year-old boy with developmental and speech delay, growth retardation, relative 
macrocephaly and dysmorphic features, which included a triangular face with frontal bossing, micrognathia, crowded teeth, low-set ears, and was suggestive of SilverRussel-like phenotype. A de novo 140-kb duplication $(6,306,154-6,446,433)$ and a de novo $586-\mathrm{kb}$ duplication $(6,690,620-7,276,764)$ in $17 \mathrm{p} 13.1$ were revealed [Coutton et al., 2012].

The third reported case was a 9-year-old girl affected by prenatal growth retardation, hypotonia, mild intellectual disability (IQ 50-58), and onset of obesity at the age of 7 years. Her face was coarse with wide palpebral fissures, upturned nostrils, and long mandible. Array-CGH analysis revealed a de novo 3.2-Mbduplication $(6,013,291-$ 9,214,914) in 17p13.1 [Kuroda et al., 2014].

The last 2 patients were reported by Mooneyham et al. [2014]. They described a 2-year-old boy with neurodevelopmental delay, macrocephaly, hypotonia, nystagmus and hyperopia. His facial features included a high anterior hairline, broad forehead, triangular face, flat occiput, broad nasal tip, full lips, openmouthed expression, and mild micrognathia. Microarray analysis identified a de novo $134-\mathrm{kb}$ duplication $(7,551,664-7,685,368)$ in chromosome $17 \mathrm{p} 13.1$ and a de novo 788 -kb duplication $(6,610,939-7,399,323)$ in chromosome 17p13.2p13.1. The second patient described was a 29 -year-old woman with mild intellectual disability, hyperactive disorder, food hoarding, seizures, hypertension, type 2 diabetes mellitus, and dyslipidemia. Her dysmorphic features included a high anterior hairline, broad nasal tip, full lips, mild micrognathia, and prominent antihelix with unfurled helix. A 62.5-kb duplication $(7,034,796-7,097,308)$ in chromosome $17 \mathrm{p} 13.1$ of unknown origin was detected [Mooneyham et al., 2014].

We made a review of the literature and compared the data of all the patients reported to date, in order to delineate a typical phenotype of the $17 \mathrm{p} 13.1$ microduplication syndrome (table 1). The 2 patients reported by Belligni et al. [2012] and Coutton et al. [2012] were re-examined in their clinical presentation; for the patients reported by Kuroda et al. [2014] and Mooneyham et al. [2014], we used the data reported in the published articles.

Intellectual disability/neurodevelopmental delay was the hallmark feature observed in all patients. The dysmorphic features in all cases were rather variable; therefore, it was difficult to delineate a common facial gestalt. Micrognathia was the most frequent facial feature in cases described so far (5/6 cases). Broad nasal tip was seen in 4 out of 6 cases, whilst rounded face, upslanting palpebral fissures, and full lips were present in half of the cases. Endocrine disorders were frequently reported, in particular obesity (4/6), dyslipidemia (4/6) and type 2 diabetes mellitus (3/6). In the patients presenting with endocrine disorders, clinical symptoms were evident as early as the second half of the first decade of life. Hyperactivity and food hoarding were seen in 4 out of 6 reported patients to date.

Three out of 6 patients showed abnormalities on brain MRI. Since all of the observed abnormalities were different, encompassing enlarged extra-axial spaces, hippocampal malrotation, reduced gyration, choroid plexus cysts, it is difficult to say whether 'brain MRI anomalies' are a typical feature of this condition.

Our patient is the first case who presented with synophrys, short hyperconvex nails, stiffness of hand joints and motor dyspraxia. These expand the spectrum of features of the 17p13.1 duplication syndrome.

The variable size of the duplicated regions and the different genes involved may explain the heterogeneous clinical presentation amongst the patients.

The exact variation of the genomic $\mathrm{CNV}$ seen in the present case has not been reported in the CNV database to date. It involves numerous genes, with the majority of them already previously noted to have a significant role in the 17p13.1 microduplication phenotype. Several candidate genes for intellectual disability in the $17 \mathrm{p} 13.1$ region have been identified so far, including DLG4 (discs large MAGUK scaffold protein; MIM 602887), GABARAP (GABA type A receptor associated-protein like 1; MIM 605125), FXR2 (FMR1 autosomal homolog 2; MIM 605339), NLGN2 (neuroligin 2; MIM 606479), and EFNB3 (ephrin B3; MIM 602297) [Belligni et al., 2012; Coutton et al., 2012; Kuroda et al., 2014]. Most of these genes have also been previously studied in $17 \mathrm{p} 13.1$ microdeletion syndrome, suggesting their role in the mental impairment of these patients [Krepischi-Santos et al., 2009; Komoike et al., 2010; Carvalho et al., 2014].

Mooneyham et al. [2014], comparing their 2 cases with the 2 previously reported patients, defined a common region of overlap, which included DLG4, GABARAP, and $C T D N E P 1$ as the candidate genes for intellectual disability in patients with $17 \mathrm{p} 13.1$ microduplications.

Using the UCSC genome browser (GRCh37/h19), we extended the comparison of the minimal critical region to include our case and the one reported by Kuroda et al. [2014]. We identified a region overlapping with that described by Mooneyham et al. [2014] which included 7 genes (fig. 3). Some of these genes have been suggested to be implicated in neurodevelopment impairment; however, the available data are still unclear. Therefore, this linkage is still to be elucidated. 

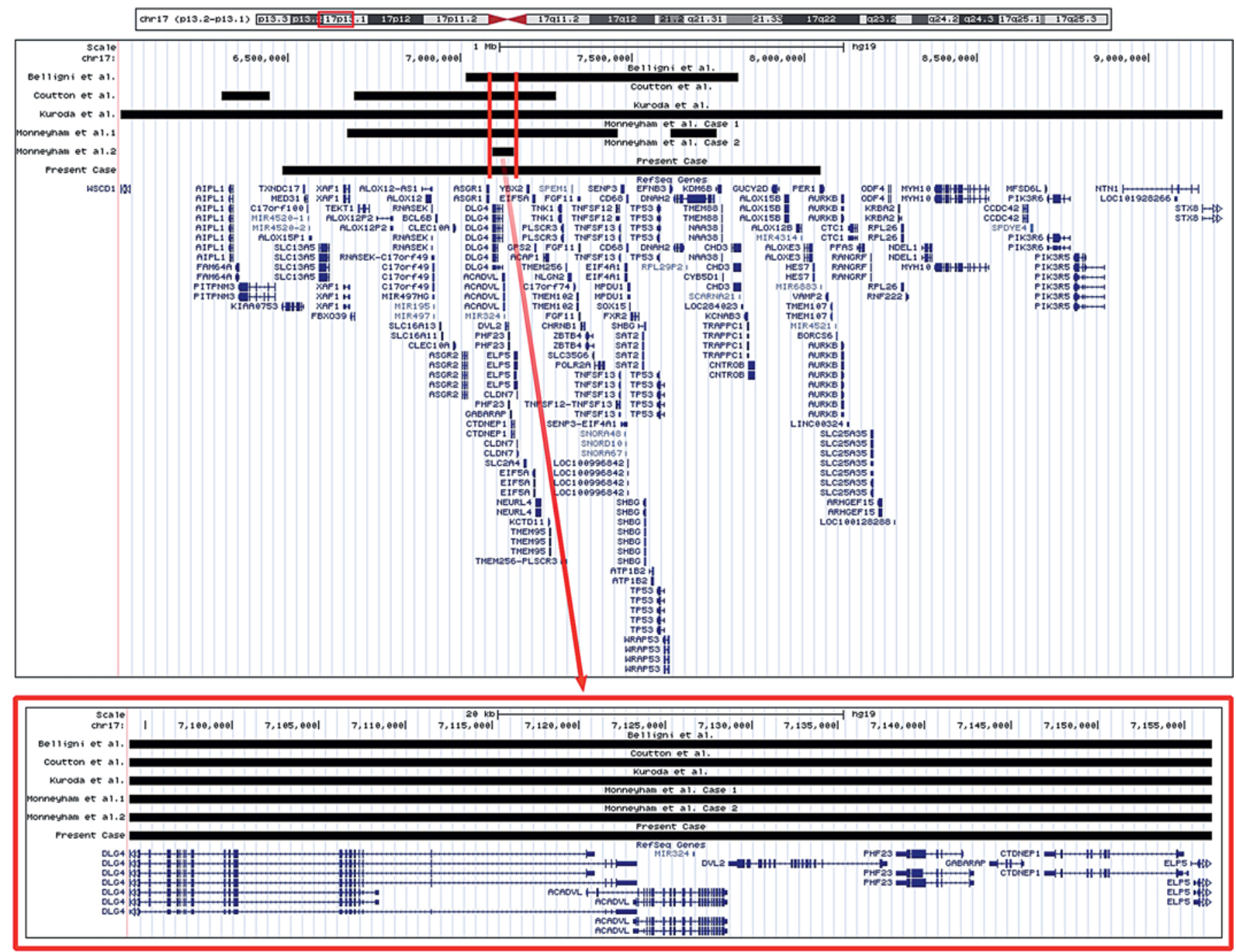

Fig. 3. The UCSC genome browser was utilized to identify the minimal region of overlap for the chromosome $17 \mathrm{p}$ duplications (chr17: 7,094,072-7,156,584 [hg19]) identified in the present case and the previous cases reported by Belligni et al. [2012], Coutton et al. [2012], Kuroda et al. [2014], and Mooneyham et al. [2014]. The horizontal black bars show the duplicated regions of patients involved. The red box (bottom) shows the 7 genes located within the minimal region of overlap.
Most of the reported duplicated regions also contain genes associated with the metabolism of carbohydrates. In particular, SLC2A4 (the insulin-responsive glucose transporter) could be involved in the $17 \mathrm{p} 13.1$ phenotype because its impaired regulation has been associated with diabetes mellitus and obesity [Belligni et al., 2012; Kuroda et al., 2014]. This gene is placed just proximal to the minimal critical region identified for the $17 \mathrm{p} 13.1$ microduplication syndrome. Both glucose intolerance and fatty liver diseases are an expression of metabolic complications of obesity; however, in these patients, as the deletion involves GLUT4, this could account for both metabolic features in addition to obesity [Kim et al., 2014].

The second case described by Mooneyham et al. [2014] affected by type 2 diabetes mellitus and obesity had a small duplicated region, corresponding to the minimal critical region, not including $S L C 2 A 4$. We propose a possible position effect to explain the woman's phenotype. To date, 4/6 patients are obese, and 3 of them developed diabetes. The remaining 2 patients have not shown signs of obesity to date. Keeping in mind that all of the reported cases with obesity became obese at a later age, it possibly could be still too early to evaluate the case reported 
by Mooneyham et al. [2014] regarding the child was 2 years old at the time of the report. We suppose that these clinical signs could become evident later in life. The patient reported by Coutton et al. [2012] presented growth deficiency since the prenatal period. Moreover, he required growth hormone treatment from the age of 4 years. Based on current knowledge, growth retardation seen in this patient could either be the part of the 17p13.1 microduplication syndrome or could be caused by some other unrecognized condition.

In conclusion, $17 \mathrm{p} 13.1$ microduplications appear to share common features such as intellectual disability, obesity, and endocrine disorders. All patients described to date had variable facial dysmorphisms; therefore, a characteristic facial gestalt was not definable. The present and previously reported cases of $17 \mathrm{p} 13.1$ microduplication do not disclose common breakpoints, but we can identify a minimal common region, whose genes are probably involved with the neurodevelopmental phenotype. Finally, we cannot ignore the presence of genes associated with metabolism as well as the endocrinological phenotypes described in most microduplicated regions reported. So we underline the need to monitor endocrinological aspects in patients with 17 p13.1 microduplication.

\section{Acknowledgments}

The authors would like to express their gratitude to the family members for their cooperation and providing the necessary medical data and photographs for publication. They would also like to thank the photographers Marco Bonazzi and Luca Valcavi. The financial support from the Fondazione Cassa di Risparmio Pietro Manodori is gratefully acknowledged.

\section{Statement of Ethics}

Informed consent was obtained from the patient's parents.

\section{Disclosure Statement}

The authors have no conflicts of interest to disclose.

\section{References}

Belligni EF, Di Gregorio E, Biamino E, Calcia A, Molinatto C, et al: $790 \mathrm{~Kb}$ microduplication in chromosome band 17p13.1 associated with intellectual disability, afebrile seizures, dysmorphic features, diabetes, and hypothyroidism. Eur J Med Genet 55:222-224 (2012).

- Carvalho CMB, Vasanth S, Shinawi M, Russell C, Ramocki MB, et al: Dosage changes of a segment at $17 \mathrm{p} 13.1$ lead to intellectual disability and microcephaly as a result of complex genetic interaction of multiple genes. Am J Hum Genet 95:565-578 (2014).

-Cole TJ, Bellizzi MC, Flegal KM, Dietz WH: Establishing a standard definition for child overweight and obesity worldwide: international survey. BMJ 320:1240-1243 (2000).
Coutton C, Devillard F, Vieville G, Amblard F, Lopez G, et al: 17p13.1 microduplication in a boy with Silver-Russell syndrome features and intellectual disability. Am J Med Genet A 158A:2564-2570 (2012).

Kim S, Jung J, Kim H, Heo RW, Yi CO, et al: Exendin-4 improves nonalcoholic fatty liver disease by regulating glucose transporter $4 \mathrm{ex}$ pression in $\mathrm{ob} / \mathrm{ob}$ mice. Korean J Physiol Pharmacol 18:333-339 (2014).

Komoike Y, Shimojima K, Liang JS, Fujii H, Maegaki $Y$, et al: A functional analysis of GABARAP on $17 \mathrm{p} 13.1$ by knockdown zebrafish. J Hum Genet 55:155-162 (2010).

-Krepischi-Santos ACV, Rajan D, Temple IK, Shrubb V, Crolla JA, et al: Constitutional haploinsufficiency of tumor suppressor genes in mentally retarded patients with microdeletions in 17p13.1. Cytogenet Genome Res 125: 1-7 (2009).
Kuroda Y, Ohashi I, Tominaga M, Saito T, Nagai JI, et al: De novo duplication of 17p13.1-p13.2 in a patient with intellectual disability and obesity. Am J Med Genet Part A 164A:15501554 (2014).

Mooneyham KA, Holden KR, Cathey S, Dwivedi A, Dupont BR, Lyons MJ: Neurodevelopmental delays and macrocephaly in $17 \mathrm{p} 13.1 \mathrm{mi}-$ croduplication syndrome. Am J Med Genet A 164A:2887-2891 (2014).

Uwaifo GI, Fallon EM, Chin J, Elberg J, Parikh SJ, Yanovski JA: Indices of insulin action, dispos$\mathrm{al}$, and secretion derived from fasting samples and clamps in normal glucose-tolerant black and white children. Diabetes Care 25:20812087 (2002). 